\title{
Weld Seam Deviation Prediction of Gas Metal Arc Welding Based on Arc Sound Signal
}

\author{
Wang Zhao, Jianfeng Yue, Wenji Liu, Haihua Liu \\ School of Mechanical Engineering, Tiangong University, Tianjin, China \\ Email: 361108443@qq.com
}

How to cite this paper: Zhao, W., Yue, J.F., Liu, W.J. and Liu, H.H. (2021) Weld Seam Deviation Prediction of Gas Metal Arc Welding Based on Arc Sound Signal. World Journal of Engineering and Technology, 9, 51-59.

https://doi.org/10.4236/wjet.2021.91004

Received: December 19, 2020

Accepted: January 18, 2021

Published: January 21, 2021

Copyright () 2021 by author(s) and Scientific Research Publishing Inc. This work is licensed under the Creative Commons Attribution International License (CC BY 4.0).

http://creativecommons.org/licenses/by/4.0/

\begin{abstract}
Weld seam deviation prediction is the key to weld seam tracking control, which is of great significance for realizing welding automation and ensuring welding quality. Aiming at the problem of weld seam deviation prediction in GMAW (gas metal arc welding), a method of weld seam deviation prediction based on arc sound signal is proposed. By analyzing the feature of the arc sound signal waveform, the time domain feature of the arc sound signal is extracted. The wavelet packet analysis method is used to analyze the time-frequency domain feature of the arc sound signal, and the wavelet packet energy feature is extracted. The time domain feature and wavelet packet energy feature are used to establish the feature vector, and the BP (back propagation) neural network is used to realize the weld seam deviation prediction. The results show that the method proposed in this paper has a good weld seam deviation prediction effect, with a mean absolute error of $0.234 \mathrm{~mm}$, which provides a new method for GMAW weld seam recognition.
\end{abstract}

\section{Keywords}

Weld Seam Deviation, GMAW, Arc Sound, BP Neural Network

\section{Introduction}

The deviation of the weld seam during the welding process will cause defects in the weld seam formation and deterioration of the mechanical properties of the welded structure. The key to solving this problem lies in the weld seam deviation prediction technology. The automatic tracking control of the welding seam is realized by the welding seam deviation prediction technology, which can avoid the deviation of the welding seam from the groove center due to the processing error and thermal deformation of the workpiece [1] [2] [3]. This can greatly improve the production efficiency of welding work, ensure welding quality, and 
realize the automation and intelligence of the welding process.

The arc sound signal has practical application value and contains a large amount of welding information [4] [5] [6]. A skilled welder can determine whether the weld seam is deviated by hearing it. It can be seen that the arc sound signal contains useful information for predicting the weld seam deviation. At present, scholars' research on arc sound signals mainly focuses on the recognition of penetration state [7] [8], the recognition of droplet transitions [9] [10], and the detection of porosity [11], but there are few researches on the correlation between arc sound signal and weld deviation. Therefore, in this paper, aiming at the problem of GMAW weld deviation prediction, the feature of the arc sound signal of different weld seam deviation conditions are analyzed, and the time domain and wavelet packet energy feature are extracted to establish feature vectors. The BP neural network is used to realize the weld seam deviation prediction.

\section{The Experimental Setup}

The schematic diagram of the experimental setup is shown in Figure 1. It consists of three modules: the welding module, the motion control module, and the signal acquisition module. The welding module includes the Fronius TPS3200 welder, VR4000 wire feeder, welding torch, and the shielding gas cylinder. In the motion control module, the welding parameters can be adjusted and the welding work platform can be controlled through the remote control. In the signal acquisition module, the synchronous controller realizes the synchronous acquisition of the arc sound signal and the torch swing signal and transmits the signal to the computer. A condenser microphone was used to pick up the arc sound signal. The sampling frequency was $20 \mathrm{kHz}$, with the pickup distance of $30 \mathrm{~cm}$.

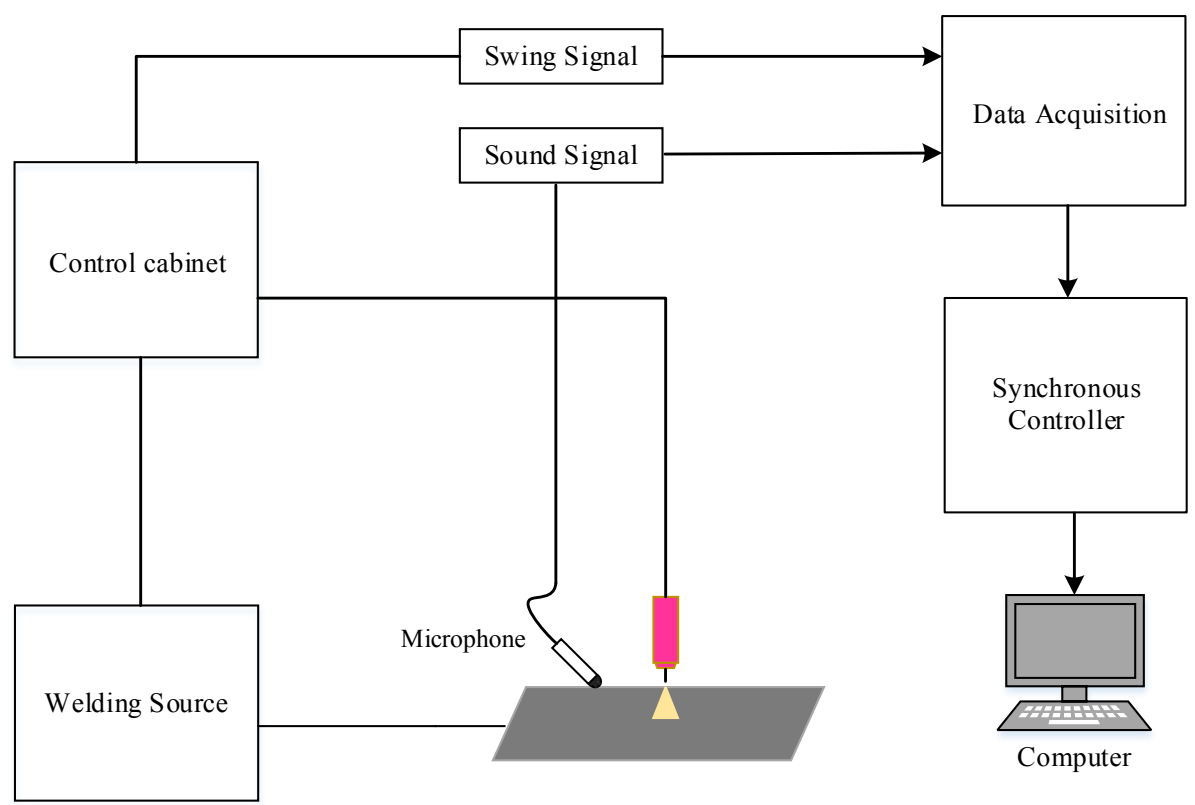

Figure 1. The experimental setup schematic diagram. 
The welding diagram and welding torch trajectory are shown in Figure 2. By controlling the distance between the welding torch swing center and the groove center, the arc sound signal of the weld seam $1 \mathrm{~mm}$ left, centered, and $1 \mathrm{~mm}$ right is obtained. Finally, the welding process parameters are shown in Table 1.

\section{Time Domain Features Extraction}

Data are collected within a welding torch swing period as a sample of arc sound signal. Figure 3 shows the arc sound signal and swing signal when the weld seam is $1 \mathrm{~mm}$ left, centered, and $1 \mathrm{~mm}$ right. When the welding torch moves from the right limit position to the left, the control cabinet sends out a high-level swing signal. On the other hand, when it moves from the left limit position to the right, a low-level signal is sent out. When the weld seam is centered, the amplitude of the arc sound signal during the right-left and left-right welding torch movements is similar. As the seam deviation increases, the arc sound signal waveform at the right and left limit position changes significantly. Therefore, the signal within $75 \mathrm{~ms}$ after the welding torch reaches the swing limit is extracted. This allows for the calculation of the energy and variance signals on both sides; the data are then subtracted to obtain the difference feature. The calculation formula is shown below.

Table 1. Welding process parameters.

\begin{tabular}{cc}
\hline Welding Parameter & The parameter value \\
\hline Welding current/(A) & 120 \\
Swing amplitude/(mm) & 6 \\
Swing frequency $/(\mathrm{Hz})$ & 1.5 \\
Wire diameter $/(\mathrm{mm})$ & 1 \\
Welding speed/(mm/s) & 3.2 \\
Shielding gas & $82 \% \mathrm{Ar}+18 \% \mathrm{CO}_{2}$
\end{tabular}

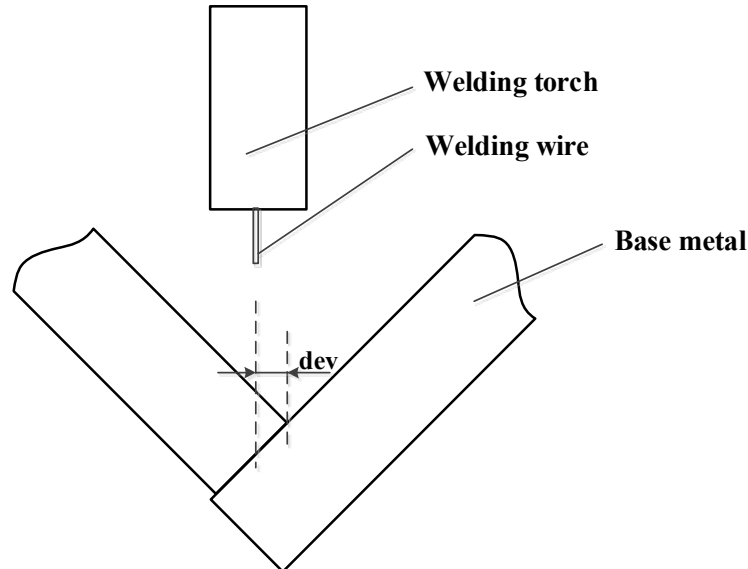

(a)

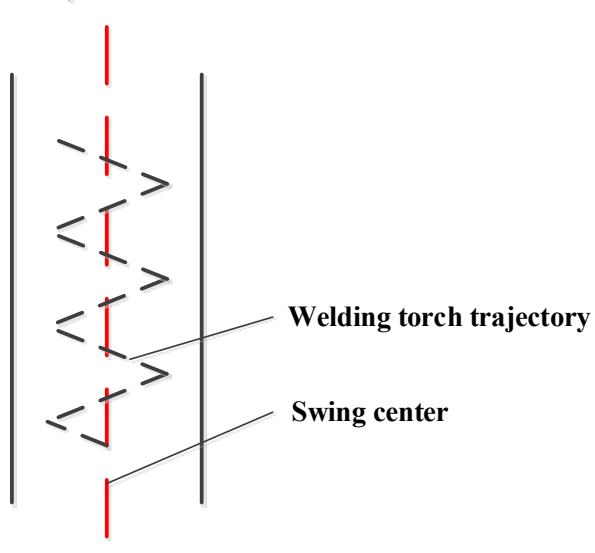

(b)

Figure 2. Experiment setup: (a) welding diagram (b) torch trajectory. 


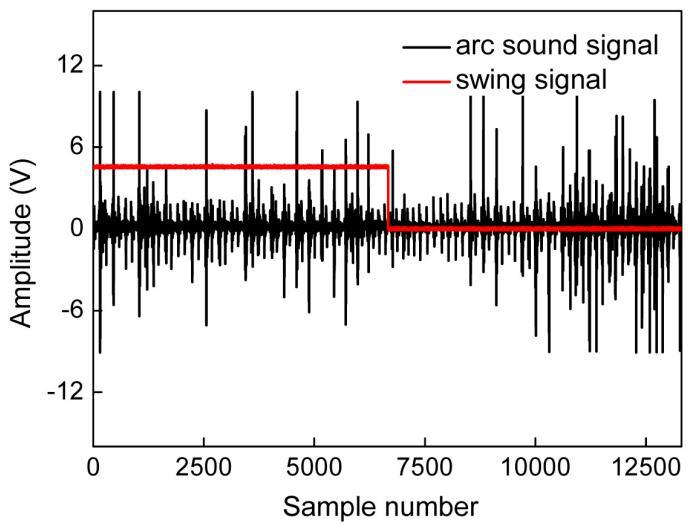

(a)

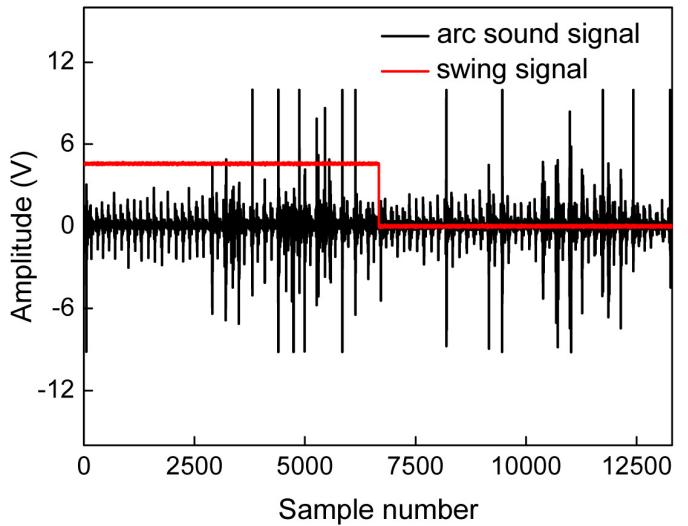

(b)

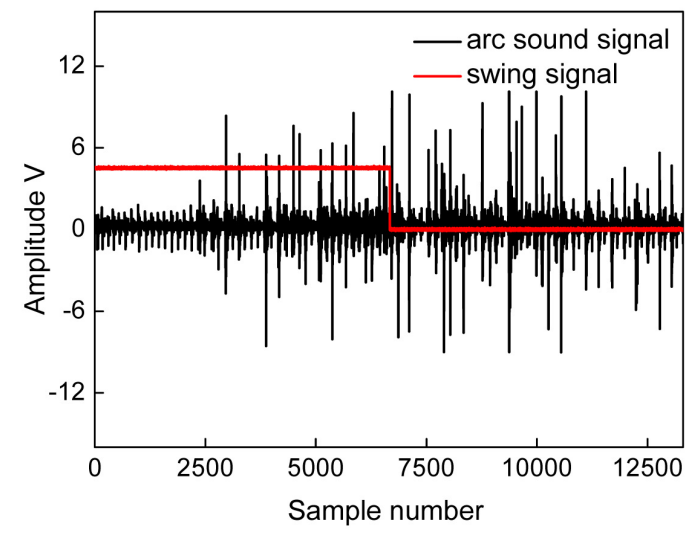

(c)

Figure 3. The comparison of arc sound signals: (a) $1 \mathrm{~mm}$ left (b) Centered (c) $1 \mathrm{~mm}$ right.

$$
\begin{gathered}
E_{d i f}=\sum_{i=1}^{n} x_{i, \text { right }}^{2}-\sum_{j=1}^{n} x_{j, \text { left }}^{2} \\
V_{d i f}=\frac{\sum_{i=1}^{n}\left(x_{i, \text { right }}-\bar{x}_{i, \text { right }}\right)^{2}}{n-1}-\frac{\sum_{j=1}^{n}\left(x_{j, \text { left }}-\bar{x}_{j, l \text { left }}\right)^{2}}{n-1}
\end{gathered}
$$

where $E_{d i f}$ represents the energy difference, $V_{\text {dif }}$ represents the variance difference, $x_{i, r i g h t}$ represents the sound pressure of the $i$-th sampling point at the right limit position, $x_{j, l \text { left }}$ represents the sound pressure of the $j$-th sampling point at the left limit position.

Figure 4 shows the time domain features of arc sound signal. It can be seen from the figure that when the weld seam is centered, the difference in arc sound energy and variance on the left and right sides is very small. When the seam is offset to the left, the energy and variance are positive, and when the seam is offset to the right, it is negative. This indicates that the deviation of the weld seam will change the time domain features of the arc sound signal.

\section{Wavelet Packet Energy Feature Extraction}

The wavelet packet analysis method is a time-frequency domain feature analysis 


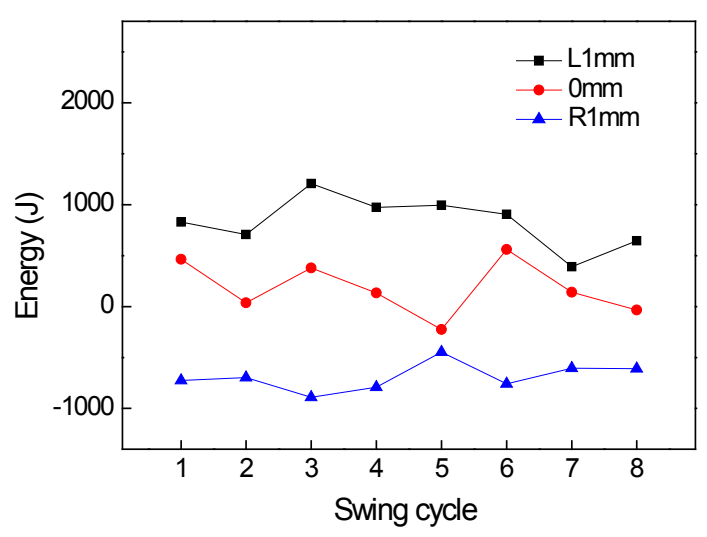

(a)

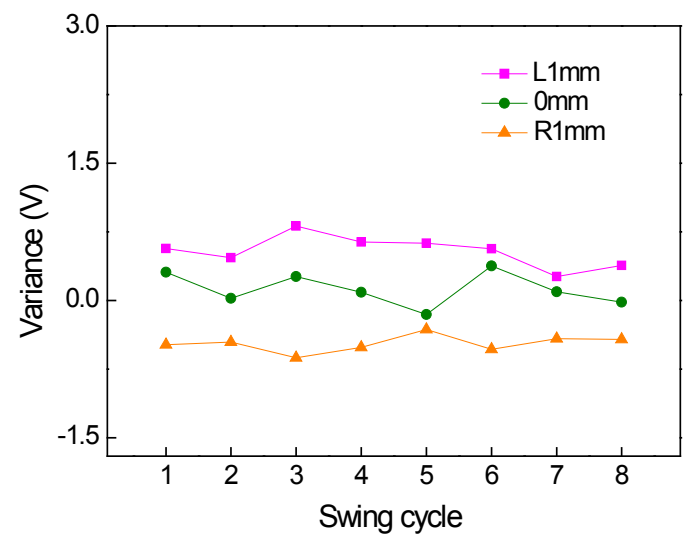

(b)

Figure 4. Time domain features of arc sound signal: (a) Energy difference (b) Variance difference.

method established on the theoretical basis of wavelet analysis. It is good at analyzing the features of non-stationary signals. It can not only decompose the low frequency band of the signal, but also decompose the high frequency band, which has strong adaptability and flexibility. The wavelet packet decomposition algorithm was written as follows [12].

$$
\begin{aligned}
& d_{l}^{j, 2 n}=\sum_{k} g_{0(k-2 l)} d_{k}^{j+1, n} \\
& d_{l}^{j, 2 n+1}=\sum_{k} g_{1(k-2 l)} d_{k}^{j+1, n}
\end{aligned}
$$

where $d_{l}^{j, 2 n}, d_{l}^{j, 2 n+1}, d_{k}^{j+1, n}$ are the wavelet packet decomposition coefficients; $g_{0(k-2 l)}$ and $g_{1(k-2 l)}$ are conjugate quadrature low-pass and high-pass filters, respectively.

The wavelet packet energy is calculated by summing up the signal squares in each frequency band (following the wavelet packet decomposition). The energy corresponding to each frequency band is shown in Equation (4):

$$
P_{i}=\sum_{k=1}^{N}\left|d_{m}^{n}(k)\right|^{2}
$$

where $N$ represents the original signal length, $d_{m}^{n}(k)$ is the $m$-th frequency band wavelet packet decomposition sequence in the $n$-th layer; $m=0,1, \cdots, 2^{n-1}$.

Daubechies3 (db3) wavelet base was selected after the comparative testing. The arc sound signal samples with $1 \mathrm{~mm}$ to the left, centering, $1 \mathrm{~mm}$ to the right of the weld seam were decomposed by three-layer wavelet packets respectively. They were divided into eight frequency bands to obtain the wavelet packet energy feature. Figure 5 shows the average values of the wavelet packet energy feature of ten sample groups with different weld seam deviation conditions. It is evident that the arc sound signal energy is primarily distributed in the high-frequency part. When there is a deviation in the welding seam, the signal energy of the first to fifth frequency bands of the arc sound signal is slightly affected. The energy of the sixth, seventh, and eighth frequency bands all decrease with the increase of the deviation. Among them, the wavelet packets energy in the seventh and eighth 


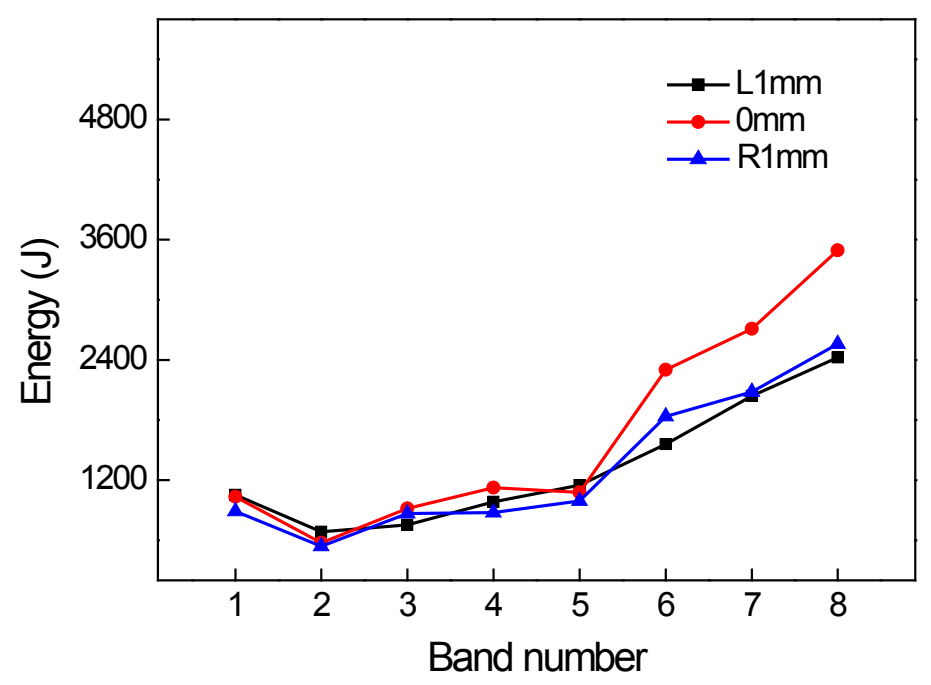

Figure 5. Wavelet packet energy feature.

frequency bands account for the largest proportion, and the energy change is symmetrical when the weld seam deviation increases. This shows that the wavelet packet energy features in the seventh and eighth frequency bands are the most stable. The wavelet packet energy feature can well reflect the deviation change of the weld seam, but it cannot reflect the deviation direction. It is necessary to combine the time domain features to jointly identify the weld seam deviation.

\section{Weld Deviation Prediction}

The four-dimensional feature vector $T=\left(E_{d i f}, V_{d i f}, P_{7}, P_{8}\right)$ is created by extracting time-domain and wavelet packet energy features. The 20 datasets were selected for each case, including the welding seam with $1 \mathrm{~mm}$ left, center, $1 \mathrm{~mm}$ right, resulting in a total of 60 data sets. From each case, 14 sets were randomly selected to form 42 training sets, and the rest as the test set. The data were normalized by mapping it to the interval $[0,1]$, mostly to improve the model convergence speed and eliminate the feature dimension influence on the model. The normalization method is shown in Equation (5):

$$
y=\frac{x-x_{\min }}{x_{\max }-x_{\min }}
$$

where $x$ represents input data, $y$ represents output data, $x_{\max }$ and $x_{\min }$ represent the maximum and minimum values of input data respectively.

BP neural network is used to establish the weld seam deviation prediction model. Set the number of input neurons to 4 , the number of hidden layer neurons to 5 , the number of output neurons to 1 , the learning rate to 0.1 , the maximum training times to 1000 , and the target error to $1 \times 10^{-5}$. BP neural network structure diagram is shown in Figure 6. The prediction result of weld seam deviation is shown in Figure 7, and the mean absolute error is $0.234 \mathrm{~mm}$. The BP neural network weld seam deviation prediction model established in this paper 


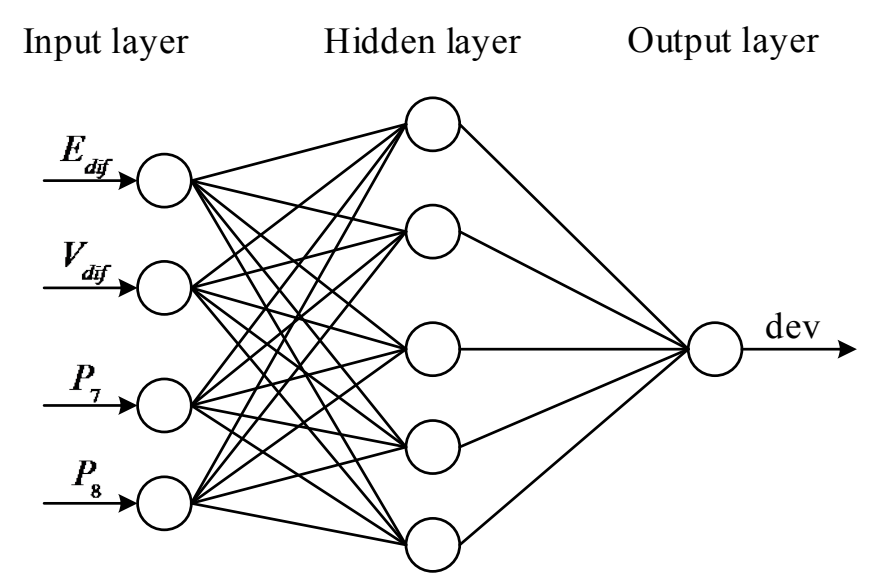

Figure 6. BP neural network structure diagram.

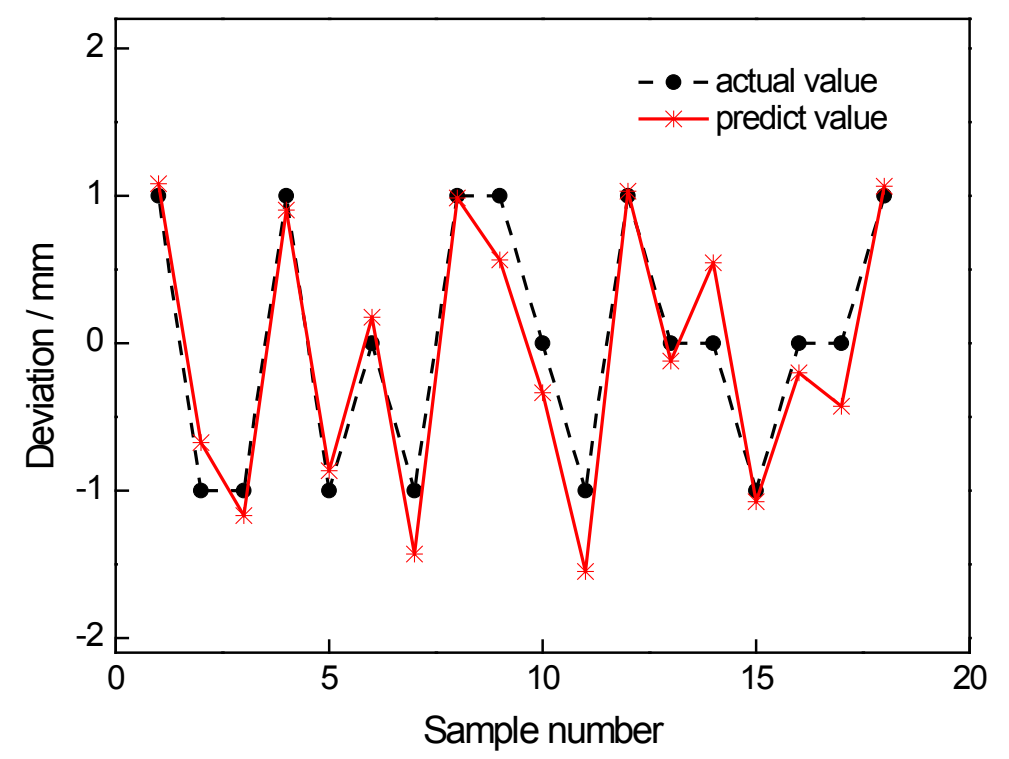

Figure 7. Results of weld seam deviation prediction.

has achieved good prediction results. It can be seen that it is feasible to use the arc sound signal to predict the deviation of the GMAW weld seam, which provides a new way for real-time tracking and control of the weld seam.

\section{Conclusions}

This paper proposes a method of weld seam deviation prediction based on arc sound signal.

When there is a deviation in the weld seam, the waveform at the limit position of the arc sound signal changes significantly, so the energy and variance difference features at the limit position are extracted. Analyzing the wavelet packet energy feature of the arc sound signal, it is found that the high-frequency band energy decreases significantly with the increase of the weld seam deviation. The seventh and eighth frequency band wavelet packet energy features with the best performance are extracted. A four-dimensional feature vector is constructed us- 
ing the time domain features and wavelet packet energy features of arc sound signals. The BP neural network is used to predict the weld seam deviation. The mean absolute error of the prediction results is $0.234 \mathrm{~mm}$, which proves the effectiveness of the weld seam deviation prediction method proposed in this paper. Future work includes optimizing the predictive model and developing a real-time control system for seam tracking based on arc sound signals.

\section{Conflicts of Interest}

The authors declare no conflicts of interest regarding the publication of this paper.

\section{References}

[1] Liang, B.Y., Xu, X.Y., Gong, S.H., Wang, Q.X. and Wang, X.R. (2016) Dual-Beam Laser Welding and Seam Tracking Control Technology for 3D T-Beam. Transactions of the China Welding Institution, 37, 47-50+131.

[2] Li, Y., Xu, D., Li, T., Wang, L.K. and Tan, M. (2005) On Visual Sensors for Welding Seam Tracking Based on Laser Structured Light. Journal of Transduction Technology, No. 3, 488-492.

[3] Park, J.H. and Moon, H.S. (2020) Advanced Automatic Welding System for Offshore Pipeline System with Seam Tracking Function. Applied Sciences, 10, 324. https://doi.org/10.3390/app10010324

[4] Saini, D. and Floyd, S. (1998) An Investigation of Gas Metal Arc Welding Sound Signature for On-line Quality Control. Welding Journal, 77, 172S-179S.

[5] Jiang, F., Li, Y.F. and Chen, S.J. (2018) Current Situation and Prospects of Welding Arc Monitoring Technology. Journal of Mechanical Engineering, 54, 16-26. https://doi.org/10.3901/JME.2018.02.016

[6] Čudina, M. and Prezelj, J. (2003) Evaluation of the Sound Signal Based on the Welding Current in the Gas-Metal Arc Welding Process. Proceedings of the Institution of Mechanical Engineers, Part C: Journal of Mechanical Engineering Science, 217, 483-494. https://doi.org/10.1243/095440603765226777

[7] Gao, Y.F., Wang, Q.S., Xiao, J.H. and Zhang, H. (2020) Penetration State Identification of Lap Joints in Gas Tungsten Arc Welding Process Based on Two Channel Arc Sounds. Journal of Materials Processing Technology, 285, Article ID: 116762. https://doi.org/10.1016/j.jmatprotec.2020.116762

[8] Cui, Y.X., Shi, Y.H., Zhu, T. and Cui, S. (2020) Welding Penetration Recognition Based on Arc Sound and Electrical Signals in K-TIG Welding. Measurement, 163, Article ID: 107966. https://doi.org/10.1016/j.measurement.2020.107966

[9] Kamal, P., Sandip, B. and Surjya, K.P. (2009) Prediction of Metal Deposition from Arc Sound and Weld Temperature Signatures in Pulsed MIG Welding. The International Journal of Advanced Manufacturing Technology, 45, 1113-1130. https://doi.org/10.1007/s00170-009-2052-5

[10] Gao, Y.F., Wang, Q.S., Huang, L.R., Gong, Y.F. and Xiao, J.H. (2019) Droplet Transfer Modes Identification in MIG Welding Process Based on a Human Auditory Model. Journal of Mechanical Engineering, 55, 68-76. https://doi.org/10.3901/JME.2019.17.068

[11] Yusof, M.F.M., Kamaruzaman, M.A., Ishak, M. and Ghazali, M.F. (2017) Porosity Detection by Analyzing Arc Sound Signal Acquired during the Welding Process of 
Gas Pipeline Steel. International Journal of Advanced Manufacturing Technology, 89, 3661-3670. https://doi.org/10.1007/s00170-016-9343-4

[12] Gao, S., Wu, Y.N. and Jiang, Z.H. (2019) Static and Dynamic Rubbing Positions Identification of Cryocooler Based on Wavelet Packet Analysis and Support Vector Machine. Journal of Infrared and Millimeter Waves, 38, 627-632. 